\title{
Ventricular Septal Rupture after Acute Coronary
}

\section{Syndrome}

\author{
Pedro Rafael de Oliveira Nascimento ${ }^{1}$, Gustavo Henrique Belarmino Góes ${ }^{* 2}$, Caroline Bernardi Fabro ${ }^{2}$, \\ Mateus Lopes Barreto de Sousa ${ }^{2}$, Diana Patricia Lamprea Sepulveda ${ }^{3}$, Isly Maria Lucena de Barros ${ }^{4}$, \\ Jose Maria Del Castillo ${ }^{5}$, José Breno de Sousa Filho ${ }^{6}$, Ricardo de Carvalho Lima ${ }^{7}$, Dário Celestino Sobral Filho ${ }^{8}$ \\ ${ }^{1}$ Physician at the Messejana Hospital Dr. Carlos Alberto Studart Gomes, Fortaleza, Brazil; [pedrorafaelon@yahoo.com.br] \\ ${ }^{2}$ Medical Student at the Faculty of Medical Sciences, University of Pernambuco, Recife, Brazil. Cardiovascular Emergency Room \\ of Pernambuco (PROCAPE / University of Pernambuco), Recife, Brazil; [gustavogoesmt@ gmail.com, \\ fabro.caroline@hotmail.com,m-teuslopes@hotmail.com] https://orcid.org/0000-0003-3366-1182 \\ ${ }^{3}$ Chief of the Ward of Valvular Diseases, Preceptor and Vice-Coordinator in the Cardiology Medical Residency of the \\ Cardiovascular Emergency Room of Pernambuco (PROCAPE / University of Pernambuco), Recife, Brazil. Master in Cardiology \\ (University of Pernambuco); [lampreadiana@gmail.com] https://orcid.org/0000-0001-8055-2128 \\ ${ }^{4}$ Member of the Post-Graduation and Research Center of the Oswaldo Cruz University Hospital and Physician in the Ward of \\ Coronary Diseases of the Cardiovascular Emergency Room of Pernambuco (PROCAPE / University of Pernambuco), Recife, \\ Brazil. Doctorate in Medicine (University of São Paulo); [isly.lucena@ @mail.com] \\ ${ }^{5} \mathrm{CEO}$ of the School of Ultrasound of Pernambuco (Ecope); Voluntary Professor of Specialization in Echocardiography of the \\ Cardiovascular Emergency Room of Pernambuco (PROCAPE / University of Pernambuco). Vice-President of Echocardiography \\ of the Department of Cardiovascular Image of the Brazilian Society of Cardiology, Recife, Brazil. Doctorate in Medicine \\ (National University of La Plata); [castillojmd@gmail.com] \\ ${ }^{6}$ Founding Member of the Latin-American Society of Interventional Cardiology, Assistant Professor at the University of \\ Pernambuco, and Head of the Hemodynamics Laboratory of the Cardiovascular Emergency Room of Pernambuco (PROCAPE / \\ University of Pernambuco), Recife, Brazil; [jbreno50cardio@ hotmail.com] https://orcid.org/0000-0003-4040-3296 \\ ${ }^{7}$ Associate Professor of Cardiology at the University of Pernambuco, Executive Director of the Cardiovascular Emergency Room \\ of Pernambuco (PROCAPE / University of Pernambuco). Head of the Cardiovascular Surgery Team of the Cardiovascular \\ Emergency Room of Pernambuco, Recife, Brazil. Doctorate in Medicine (Federal University of São Paulo); \\ [rclima@elogica.com.br] https://orcid.org/0000-0002-1369-0296 \\ ${ }^{8}$ Associate Professor of Cardiology at the University of Pernambuco. Research Coordinator of the Cardiovascular Emergency \\ Room of Pernambuco (PROCAPE / University of Pernambuco), Recife, Brazil. Doctorate in Cardiology (Federal University of \\ Rio Grande do Sul). Fellow of the American College of Cardiology and European Society of Cardiology; [dsobral@uol.com.br] \\ https://orcid.org/0000-0002-5301-7741
}

Corresponding author: Gustavo Góes; gustavogoesmt@gmail.com; https://orcid.org/0000-0003-3366-1182

Received 13 August 2019;

Accepted 15 October 2019;

Published 21 October 2019

\begin{abstract}
Objective: Ventricular septal rupture (VSR) is a rare but serious complication of acute myocardial infarction, which occurs in about 0.2 to $0.3 \%$ of patients with myocardial ischemia. If early therapy is not initiated, 90\% of patients with VSR will die within the first month. This study aimed to evaluate the epidemiological and clinical characteristics of patients with VSR as a mechanical complication of acute myocardial infarction. Methods: A prospective study was conducted among nine patients who presented to the Cardiovascular Emergency Room of Pernambuco with acute coronary syndrome with ST segment elevation and VSR complications. Results: There were five women and 4 men, and the mean age of the patients was 72.5 years. The median time from the onset of the symptoms of acute coronary syndrome with ST segment elevation to the diagnosis of VSR was 3.5 days. Among the nine patients included in the study, three were treated surgically. Of all the patients, including those who underwent corrective surgery, eight patients died, $44.4 \%(\mathrm{~N}=4)$, in the first four days after AMI. Conclusion: VSR occurs more frequently among elderly patients with multi-arterial involvement, lower wall infarction, and involvement of the right coronary artery. The prognosis is extremely limited, especially in patients who are already admitted to the cardiac emergency room with Killip IV, with > 24 hours of clinical evolution, and do not require surgical correction.
\end{abstract}

Keywords: Ventricular septal rupture; acute coronary syndrome with ST segment elevation; mechanical complication 


\section{Introduction}

Ventricular septal rupture (VSR) is a rare but serious complication of acute myocardial infarction (AMI), and the responsible artery of which is the right coronary artery. ${ }^{[1]}$ It is estimated that this complication occurs in about 0.2 to $0.3 \%$ of patients with AMI. ${ }^{[2,3]}$ If no early therapeutic approach is initiated, $90 \%$ of patients with VSR will die within the first month. The surgical approach is associated with a higher survival rate of around 55\%. ${ }^{[4,5]}$

The prognosis of the patient is directly related to the early diagnosis and initiation of the intervention, which occurs in the first 24 hours of acute ischemic episode on average. ${ }^{[6]}$

There are several disagreements in the literature on the utilization of the most appropriate approach for the management of VSR. Thus, this study described a series of nine consecutive patients who presented with VSR caused by AMI and analyzed the epidemiology, initial clinical evaluation, complementary investigation, therapeutic approaches adopted, and outcome.

\section{Methods}

This is a prospective, descriptive, case series study of nine patients who were admitted to the Cardiovascular Emergency Room of Pernambuco (PROCAPE / University of Pernambuco) and diagnosed with acute coronary syndrome with ST elevation and VSR. To be included in this series, patients should have this diagnosis confirmed by some complementary methods (transthoracic echocardiography or cardiac catheterization).

Epidemiological analysis (age, sex), clinical evaluation (personal history, physical examination findings, Killip classification, clinical or surgical treatment), and complementary exams (electrocardiography, echocardiography, hemodynamic study) were performed. Likewise, the clinical outcome was analyzed.

\section{Statistical analysis}

Continuous variables with normal distribution were presented as means \pm standard deviation, and those with non-normal distribution were presented as medians. Nominal variables were expressed as absolute and percentage numbers.

\section{Results}

\section{Clinical features}

Table 1 shows the patients' baseline demographic data and clinical characteristics. The mean age was 72.5 years, and the patients were five females and four males. Most patients $(\mathrm{N}=6)$ had systemic arterial hypertension, and four of them were diabetic. The presence of smoking was observed in four patients, while none of the patients had a history of renal or chronic ischemic disease and AMI. The median time from the onset of symptoms of acute coronary syndrome with ST segment elevation to the diagnosis of VSR was 3.5 days (range, 1 to 11 days).

Table 1: Demographic data and clinical characteristics of patients

\begin{tabular}{|l|c|c|}
\hline Criteria & VSR (N=9) & $\%$ \\
\hline Age - yr & 73.1 & -- \\
\hline Male & 4 & 44.4 \\
\hline Arterial hypertension & 6 & 66.7 \\
\hline Diabetes mellitus & 4 & 44.4 \\
\hline Smoking & 4 & 44.4 \\
\hline Mean systolic blood pressure - $\mathrm{mmHg}$ & 100.1 & -- \\
\hline
\end{tabular}

\begin{tabular}{|l|c|c|}
\hline Mean diastolic blood pressure - mmHg & 61.0 & -- \\
\hline Male & 4 & 44.4 \\
\hline Arterial hypertension & 6 & 66.7 \\
\hline Killip classification & & \\
\hline I & 3 & 33.3 \\
\hline II & 1 & 11.1 \\
\hline III & 0 & -- \\
\hline IV & 5 & 55.6 \\
\hline
\end{tabular}

VSR: Ventricular septal rupture

Five patients presented with Killip IV classification (cardiogenic shock). Of the nine patients, four of them had a heart rate $>100$ beats per minute and five had a systolic blood pressure $\leq 100$ $\mathrm{mmHg}$. Electrocardiogram was performed among all patients; however, transthoracic echocardiogram (TTE) with Doppler was the most common complementary method for the initial diagnosis.

\section{Complementary evaluation}

An electrocardiogram was performed among all patients at the time of admission, with an equal number of cases of lower wall infarction (four patients) and previous infarction. Only one patient had a combination of anterior and inferior infarctions (Table 2).

Table 2: Electrocardiographic and echocardiographic characteristics of patients with ventricular septal rupture (VSR)

\begin{tabular}{|l|l|l|}
\hline Characteristics & VSR (N=9) & \% \\
\hline Electrocardiogram (Ischemic wall) & & \\
\hline Anterior & 4 & 44.4 \\
\hline Inferior & 4 & 44.4 \\
\hline Anterior and inferior & 1 & 11.1 \\
\hline Echocardiography (Place of rupture) & & \\
\hline Apical & 6 & 66.7 \\
\hline Basal & 1 & 11.1 \\
\hline Not determined & 2 & 22.2 \\
\hline Mean of left ventricular ejection fraction & -- & 58.6 \\
\hline
\end{tabular}

Doppler TTE was also performed in all patients. Most defects were observed in the apical portion of the septum (six cases) (Figure 1), basal portion (one case), and undetermined location (two cases). The left ventricular ejection fraction (LVEF) was only estimated in three patients. Meanwhile, the LVEF was determined in the other six patients, ranging from 34 to $67 \%$ (Table 2).

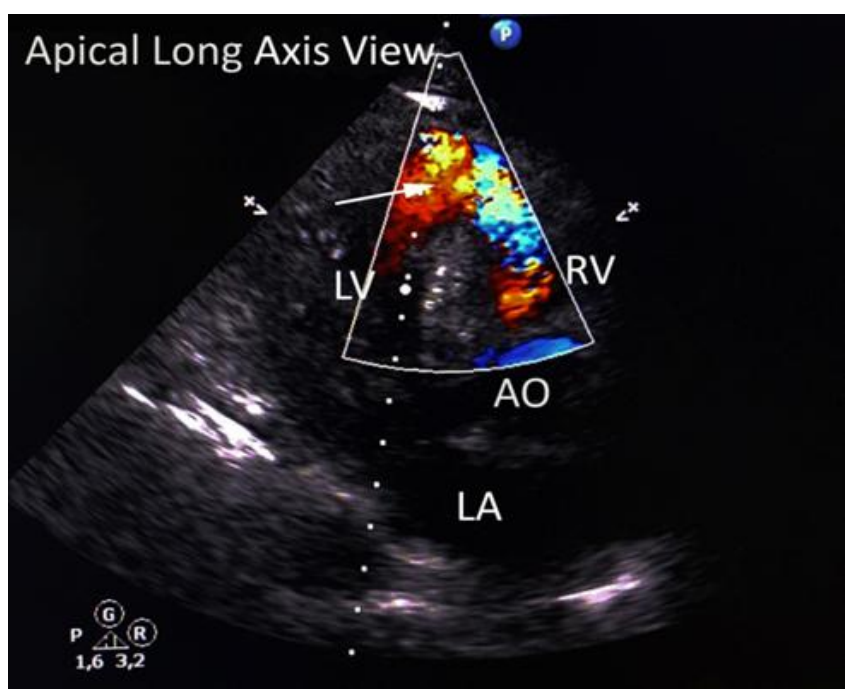

Figure 1: Two-dimensional echocardiogram due to the modified apical long axis view showing a large rupture of the ventricular septum in the apical region after myocardial infarction, with left to right flow demonstrated by color flow 
mapping. LV: left ventricle; RV: right ventricle; AO: aorta; LA: left atrium.

Cardiac catheterization was performed in eight patients: in five patients, the artery responsible for the infarction was the right coronary artery, and in three cases, the anterior descending artery (Figure 2). There was some degree of culprit artery stenosis among all patients, and in most cases $(\mathrm{N}=7)$, the artery was totally occluded. Furthermore, the results revealed a higher prevalence of multi-arterial disease, with six patients presenting with at least two epicardial vessels (Table 3).

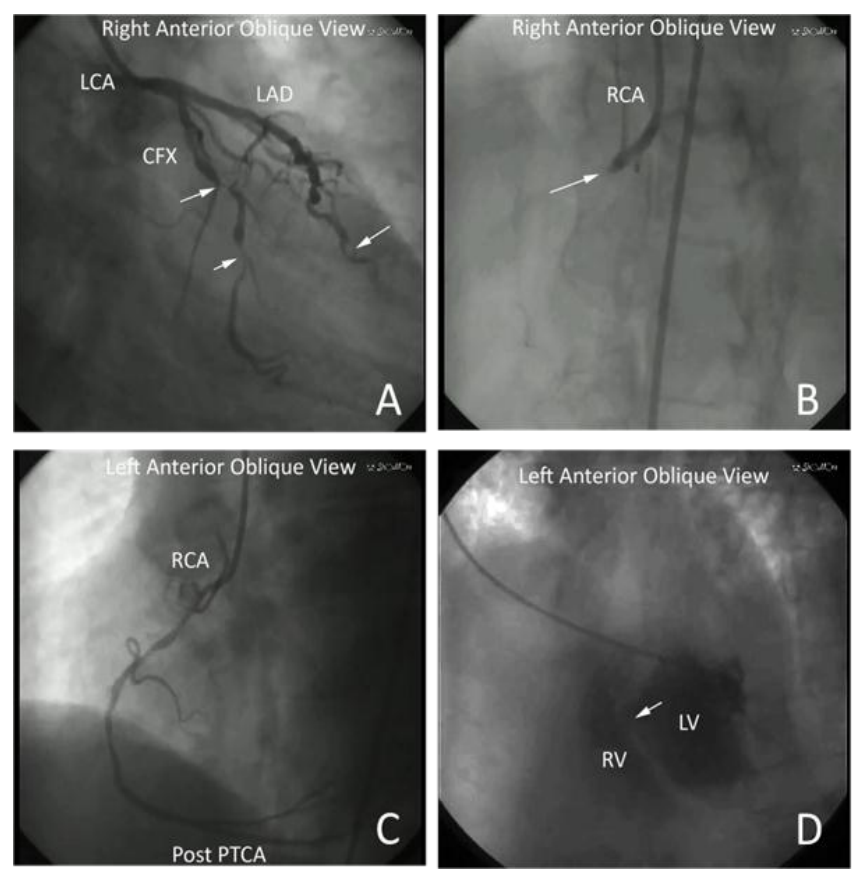

Figure 2: Cineangiography in a patient with ventricular septal rupture after myocardial infarction. A. Left coronary artery (LCA) in the right anterior oblique view showing total anterior descending branch (ADB) obstruction in the medial third and several severe lesions in the circumflex branch (CFX). B. Right coronary artery (RCA) in the right anterior oblique view showing total obstruction in its initial third. C. RCA in the left anterior oblique view recanalized after percutaneous angioplasty. $D$. Left ventricular cineventriculography in the left anterior oblique view showing right ventricular filling through rupture of the interventricular septum. The arrows indicate the changes. LV: left ventricle; RV: right ventricle.

Table 3: Angiographic characteristics of patients with ventricular septal rupture (VSR)

\begin{tabular}{|l|l|l|}
\hline Characteristics & VSR (N=8) & $\%$ \\
\hline Affected related artery & & \\
\hline Anterior descending artery & 3 & 37.5 \\
\hline Right coronary artery & 5 & 62.5 \\
\hline Circumflex artery & 0 & -- \\
\hline Number of vessels affected & & \\
\hline 1 & 2 & 25.0 \\
\hline 2 & 3 & 37.5 \\
\hline 3 & 3 & 37.5 \\
\hline Affected artery stenosis & 8 & 100.0 \\
\hline
\end{tabular}

Treatment, complications, and outcome

Of the nine patients, only three came in a timely manner to perform cardiac catheterization with subsequent angioplasty, one of them being a rescue (patient previously received an injectable tenecteplase but did not meet the reperfusion criteria), and the remaining patients already had an evolution $>24$ hours. One of the patients who presented with lower wall acute coronary syndrome with ST elevation, arrived with > 24 hours evolution, was submitted for invasive stratification that showed multi-arterial disease with severe lesions in the anterior descending artery and first diagonal branch, and with a totally occluded right coronary artery was chosen for percutaneous treatment of the anterior descending with a pharmacological stent.

Inotropic therapy with dobutamine was necessary for all patients. Norepinephrine was administered in seven patients and vasodilators were used in two patients. Cardiogenic shock was observed in seven patients while five patients had Killip IV classification. Of the nine patients, five $(55.6 \%)$ of them had an intra-aortic balloon pump, always associated with inotropic therapy. Seven patients $(77.8 \%)$ required mechanical ventilation and one presented with ventricular fibrillation. Another common complication was acute renal failure, which occurred in eight patients (Table 4).

Table 4: Treatment performed, in-hospital procedures, medication use, and outcome

\begin{tabular}{|l|c|c|}
\hline Characteristics & VSR (N=9) & \% \\
\hline Treatment & & \\
\hline VSR surgical correction & 3 & 33.3 \\
\hline Clinical & 6 & 66.7 \\
\hline In-hospital approach & & \\
\hline Intra-aortic balloon & 5 & 55.6 \\
\hline Mechanical ventilation & 7 & 77.8 \\
\hline Use of medications & & \\
\hline Vasodilators & 2 & 22.2 \\
\hline Norepinephrine & 9 & 100.0 \\
\hline Renal dysfunction & 8 & 88.9 \\
\hline Cardiogenic shock & 7 & 77.8 \\
\hline
\end{tabular}

VSR: Ventricular septal rupture

Of the nine patients, surgery was performed in three patients: two of them underwent VSR correction alone and the other underwent VSR correction with myocardial revascularization surgery of the anterior descending artery.

Of the three surgical approaches to the defect, one approach was performed five days after the diagnosis of VSR and suspension of the double platelet antiplatelet regimen with aspirin and clopidogrel. The patient's hemodynamic stability was another factor contributing to the delay of the surgical correction.

Of the three patients, two developed complications after the procedure: one patient had VSR with hemodynamic repercussion and the other patient developed cardiac tamponade. Of all patients, including those who underwent corrective surgery, eight died, $44.4 \%(\mathrm{~N}=4)$, in the first four days after AMI (range, 2 to 44 days). Progressive heart failure was the most common complication (Table 4).

\section{Discussion}

Post-AMI VSR is more prevalent in women with advanced age and previous episode of cerebral ischemic stroke. Chronic kidney disease is more common in these patients. On the other hand, due to collateral circulation caused by previous coronary artery disease, these individuals are outside the group of risk factors for heart disease, such as smoking and hypertension. ${ }^{[1,6]}$ About seven patients $(77.8 \%)$ were hypertensive, four $(44.4 \%)$ were diabetic, and four $(44.4 \%)$ were smokers. The main consequences of VSR are related to the hemodynamic changes of the cardiac cycle, with 
congestive heart failure and cardiogenic shock as the main problem. ${ }^{[4,7,8]}$ The latter occurred in $77.8 \%(\mathrm{~N}=7)$ of the patients.

Accurate and safe diagnosis of post-AMI VSR is performed through percutaneous coronary intervention using the pulmonary artery balloon catheter insertion technique to document the shunt from the left to the right ventricle. In these patients, a mixed venous saturation of $\mathrm{O} 2$ is identified as a reflex of the interventricular connection. ${ }^{[1]}$

This defect is also often diagnosed by two-dimensional TTE with color Doppler for precise identification of the interventricular connection, calculation of the flow and specification of the hemodynamic repercussion of the failure, and concomitant evaluation of possible cardiac repercussions (i.e., acute heart failure). In certain circumstances, studies have shown that transesophageal echocardiography (TEE) is necessary to identify the full extent of the lesion. ${ }^{[6]}$ This is because TEE is more accurate in determining possible dilation of the right ventricle, which is an important clue to the diagnosis. In addition, this method is useful for assessing the anatomical size of the defect especially when the patient has bad acoustic windows due to the mechanical ventilation. Because of its greater sensitivity for evaluation of the defect size, TEE is used to guide the closure of the lesion through a percutaneous or surgical approach. ${ }^{[1,9]}$

The echocardiogram and catheterization evaluation, time between AMI and diagnosis, and presence of Killip IV (cardiogenic shock) indicate worsening of the patient's clinical condition, leading to death in a few days, as presented in the cases analyzed.

The evidence of high mortality in cases of post-AMI VSR stands out with the $88.89 \%$ mortality rate in nine patients prospectively analyzed, even those who underwent surgical repair. The therapy involved in VSR is still limited by external factors, such as time, with a better prognosis when the diagnosis and conduct are taken within 24 hours of the beginning of AMI. For an effective approach, it is necessary to evaluate each case individually because of the size of the interventricular shunt, which may be severe enough to cause a patient to crash or only cause a minor regurgitation. In the first case, surgical intervention is imperative to increase the patient's chance of survival; however, in the second case, the waiting time to undergo surgery and have a better prognosis is six weeks, since healing of the affected area will already be stable and will make the intervention safer. ${ }^{[6]}$

A delay in the surgical approach may be selected for hemodynamically stable patients undergoing double antiplatelet regimen. Thus, correction of the defect can be delayed by the time required for suspension of the antiplatelet effect. ${ }^{[10]}$ The emergency approach consists of the reperfusion of the coronary arteries with thrombolytic agents and coronary angiography as the first measures, added by the operation performed with standard cardiopulmonary bypass by exclusion of infarction. In this surgery, an incision is made to expose the location of the VSR. The bovine pericardium is placed in the form of the ventricular infarction to identify the margins of the infarction. Then, a portion of the healthy interventricular septum is sutured intermittently with a line 3-0 polypropylene suture, as showed in Figure 3. The myocardium is closed after repair with 2-0 prolene suture. Furthermore, other possible approaches can be used, such as percutaneous transcatheter and hybrid closure, mechanical assistance (especially in acute conditions), percutaneous and left ventricular assist device, ventricular assist bioavailability device, and heart transplant. ${ }^{[7]}$
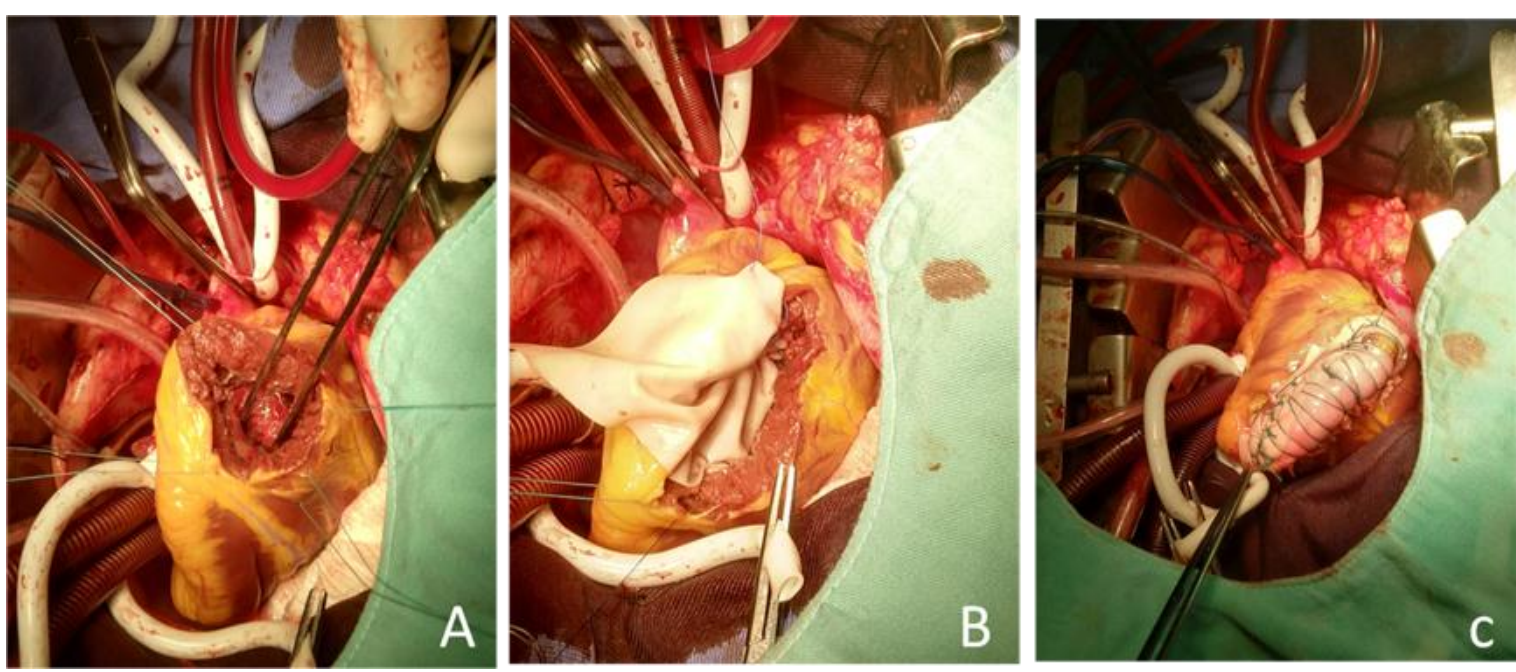

Figure 3: A. Left ventriculotomy with a septal defect of approximately $15 \mathrm{~mm}$. B. Closure of the ventricular septal defect with bovine pericardium graft. C. Final result of ventriculotomy, with the ventricular suture anchored in bovine pericardial graft.

Thus, due to the high mortality rate of surgical closure and inoperable cases, percutaneous transcatheter closure of post-AMI VSR was recommended as a less invasive therapeutic modality that can be used as a definitive intervention, therapeutic bridge for subsequent surgical correction, or procedure for the correction of residual shunts after the surgical approach. ${ }^{[8]}$ Therefore, a review of 13 case series involving 273 patients treated with percutaneous closure found an overall success rate of $89 \%$ and a 30-day or inhospital mortality of $32 \%$. ${ }^{[1]}$

Accordingly, it is necessary to identify VSR as soon as possible to assess the most effective approach and to reduce the current mortality rate of the affected patients, which has not yet significantly progressed. New types of approach and faster ways to diagnose, such as noninvasive tests, need to be studied to achieve this result.

\section{Conclusion}

Ventricular septal rupture is an uncommon complication of acute myocardial infarction which occurs more frequently among elderly patients with multivessel involvement, lower wall infarction, and right coronary artery involvement. The most frequent site of rupture is the apical region. The prognosis is extremely limited, especially in patients who are already admitted to the cardiac emergency room with Killip IV, with > 24 hours of clinical evolution, and are not for surgical correction. 


\section{Conflicts of interest}

The authors declare no conflicts of interest.

\section{Funding source}

The present study had no external funding sources.

\section{Academic linkage}

There is no linkage of this study to graduate programs.

\section{Glossary of abbreviations}

VSR: ventricular septal rupture

AMI: acute myocardial infarction

TTE: transthoracic echocardiogram

LVEF: left ventricular ejection fraction

TEE: transesophageal echocardiography

\section{References}

1. Jones BM, Kapadia SR, Smedira NG, Robich M, Tuzcu EM, Menon V, et. al. Ventricular septal rupture complicating acute myocardial infarction: a contemporary review. Eur Heart J. 2014;35(31):2060-8.

2. Heiberg J, Hjortdal VE, Nielsen-Kudsk JE. Long-term outcome after transcatheter closure of postinfarction ventricular septal rupture. J Int Cardiol. 2014;27(5):50915.

3. Huang SM, Huang SC, Wang $\mathrm{CH}, \mathrm{Wu} \mathrm{IH}$, Chi NH, Yu $\mathrm{HY}$, et al. Risk factors and outcome analysis after surgical management of ventricular septal rupture complicating acute myocardial infarction: a retrospective analysis. J Cardiothoracic Surg. 2015;10(1):66.

4. Moreyra AE, Huang MS, Wilson AC, Deng Y, Cosgrove NM, Kostis JB, et al. Trends in incidence and mortality rates of ventricular septal rupture during acute myocardial infarction. Am J Cardiol. 2010;106(8):1095100.

5. Takahashi H, Arif R, Almashhoor A, Ruhparwar A, Karck M, Kallenbach K. Long-term results after surgical treatment of postinfarction ventricular septal rupture. Eur J Cardiothorac Surg. 2015;47(4):720-4.

6. Crenshaw BS, Granger CB, Birnbaum Y, Pieper KS, Morris DC, Kleiman NS, et al. Risk factors, angiographic patterns, and outcomes in patients with ventricular septal defect complicating acute myocardial infarction. Circulation. 2000;101(1):27-32.

7. Cinq-Mars A, Voisine P, Dagenais F, Charbonneau É, Jacques F, Kalavrouziotis D, et al. Risk factors of mortality after surgical correction of ventricular septal defect following myocardial infarction: retrospective analysis and review of the literature. Int $\mathrm{J}$ Cardiol. 2016;206:27-36.

8. Tang L, Fang Z, Hu X, Tang J, Shen X, Lu X, et al. Nonsurgical repair of ventricular septal rupture after acute myocardial infarction. Int J Cardiol. 2015;185:328-32.

9. Arisha MJ, Hsiung MC, Nanda NC, Serkan B, Ahmad A, Elkaryoni A, et al. Incremental value of live/real time three-dimensional transesophageal echocardiography in the assessment of ventricular septal rupture following acute myocardial infarction. Echocardiography. 2017;34(11):1680-6.

10. Ibanez B, et al. 2017 ESC Guidelines for the management of acute myocardial infarction in patients presenting with ST-segment elevation. Eur Heart J. 2018;39(2):119-77.

11. Schlotter F, de Waha S, Eitel I, Desch S, Fuernau G, Thiele H. Interventional post-myocardial infarction ventricular septal defect closure: a systematic review of current evidence. EuroIntervention. 2016.17;12(1):94102. 